\title{
Preload from Tightening and Removal Torque
}

\author{
D. P. Hess
}

Submitted: 14 July 2019/Accepted: 8 August 2019/Published online: 23 August 2019

(C) ASM International 2019

\begin{abstract}
This paper presents a novel approach to determine preload in a bolted joint at installation or point of use. This approach uses both tightening torque and removal torque measurements. This work reveals preload is proportional to tightening torque minus removal torque and that taking this torque difference subtracts out friction. Tests with bolts, nuts, lock nuts, inserts and locking inserts are provided to assess this approach, and the results show good agreement with measured preload. The tests reveal the uncertainty in preload determined from this torque difference is less than the uncertainty in preload from tightening torque alone. A useful application of this work is to obtain a desired preload with nothing more than fastener thread pitch and a torque wrench to measure tightening torque and removal torque. This application is illustrated with data. The significance of this application is that preload is determined without any previously acquired torquetension data as needed with tightening torque alone to achieve preload. In addition, this paper presents analysis and test data for quantifying self-loosening and primary locking in threaded fasteners from tightening and removal torque measurements.
\end{abstract}

Keywords Preload · Fastener · Bolt · Nut · Insert . Tightening $\cdot$ Removal $\cdot$ Torque

D. P. Hess $(\bowtie)$

Department of Mechanical Engineering, University of South Florida, 4202 E. Fowler Avenue, ENB 118, Tampa, FL 33620, USA

e-mail: hess@usf.edu

\section{Introduction and Background}

Threaded fasteners continue to find widespread use in structures, machinery and mechanisms. In many cases, their function is to provide preload within a joint. The most common method for introducing preload in a bolted joint is through control (i.e., application and measurement) of tightening torque to the fasteners with a torque wrench [1-4]. A designer specifies a tightening torque, based on previously acquired torque-tension data, for a fastener in a given joint to achieve a desired preload within a range dictated by the uncertainty in preload resulting from using tightening torque control. This uncertainty in preload is specified [2] as plus and minus $35 \%$ for unlubricated or asreceived fasteners. It drops to plus and minus $25 \%$ for lubricated fasteners, i.e., with lubrication applied at the thread interface and nut face (or bolt face when using inserts). Alternatively, the uncertainty can be determined from torque-tension testing of sample hardware and statistical analysis [2]. Sample hardware includes the same fasteners, lubricants, assembly hardware and installation process as used in the application assembly.

The nominal preload, $F_{\mathrm{p}}$, is defined in terms of installation tightening torque, $T_{\mathrm{t}}$, as

$F_{\mathrm{p}}=\frac{T_{\mathrm{t}}}{k D}$

Here, $k$ is the nut factor determined from torque-tension data and $D$ is the nominal bolt diameter. The minimum and maximum preload are determined from the nominal preload, $F_{\mathrm{p}}$, and the uncertainty, $\Gamma$,

$$
\begin{aligned}
& F_{\mathrm{p} \min }=(1-\Gamma) F_{\mathrm{p}} \\
& F_{p \max }=(1+\Gamma) F_{\mathrm{p}}
\end{aligned}
$$

The uncertainty is specified or determined from torquetension data. Equations for minimum and maximum 
preload often include addition parameters to account for preload variation due to relaxation, creep and temperature change [1-3].

As an example, a designer specifies a tightening torque of $60 \mathrm{in}-\mathrm{lb}$ for $0.25-28$ fasteners without lubricant to achieve a nominal preload of $900 \mathrm{lb}$ with uncertainty of plus and minus $35 \%$. This means that a tightening torque of 60 in-lb will provide a preload between the minimum preload of $585 \mathrm{lb}$ (i.e., 900 minus 35\%) and the maximum preload of $1215 \mathrm{lb}$ (900 plus 35\%). The design process must check that the minimum preload is sufficient to hold the joint together and that the maximum preload is not too large to damage the fasteners or joint. This example illustrates why some designers use a nominal preload of $65 \%$ yield strength of the bolt. Namely, the maximum preload from nominal preload plus $35 \%$ uncertainty is at $100 \%$ yield strength of the bolt.

Less common methods to achieve preload are based on control of turn angle or bolt stretch. These provide reduced uncertainty, but require the use of less common and more expensive equipment, compared to a torque wrench, as well as additional training, technique and preparation.

The uncertainty in preload when using turn-angle measurement for preload is specified [2] as plus and minus $25 \%$ or as determined from turn-angle testing of sample hardware and statistical analysis.

Bolt stretch measurement is performed using special calipers or micrometer, ultrasound equipment or strain gages. These all require additional preparation of fasteners such as machining bolt ends or strain gage attachment and instrumentation. The uncertainty in preload when using bolt stretch measurement for preload is specified [2] as plus and minus $10 \%$ or as determined from testing of sample hardware and statistical analysis.

Of the three methods listed, tightening torque has the advantage of requiring common tools, techniques and training to implement, but has the highest uncertainty, whereas turn-angle and bolt stretch methods provide reduced uncertainty, but are generally more expensive and require additional tools, preparation, technique and training.

This paper presents a novel approach to determine preload from torque. Instead of using tightening torque alone with existing torque-tension data to achieve preload, it uses both tightening torque and removal torque measurements at installation. No existing torque-tension data is needed. Preload is found to be proportional to tightening torque minus removal torque. The approach is based on torque equations. Test data are provided and show the approach to be viable with reduced uncertainty compared to using tightening torque alone. Reasons for reduced uncertainty are provided.

\section{Preload from Torque Difference}

The tightening torque for a threaded fastener in a bolted joint is generally $[3,5,6]$ defined as

$T_{\mathrm{t}}=F_{\mathrm{p}}\left(\frac{p}{2 \pi}+\frac{\mu_{\mathrm{t}} r_{\mathrm{t}}}{\cos \beta}+\mu_{\mathrm{n}} r_{\mathrm{n}}\right)$

Here, $T_{\mathrm{t}}$ is the tightening torque, $F_{\mathrm{p}}$ is the preload, $p$ is the thread pitch, $\mu_{\mathrm{t}}$ is the thread interface friction coefficient, $r_{t}$ is the nominal thread interface radius, $\beta$ is the thread half angle, $\mu_{\mathrm{n}}$ is the nut face friction coefficient, and $r_{\mathrm{n}}$ is the nominal nut face radius. The first term in this equation is the torque required to stretch the bolt, and the remaining two terms are the torque required to overcome thread and nut friction.

Using torque-tension data, tightening torque is typically specified and applied with a torque wrench to achieve desired preload. Equation 3 provides a relation between preload and tightening torque. The two coefficients of friction are the most difficult to estimate. Friction introduces the most uncertainty in this relation and in torquetension data. In practice, this translates to uncertainty in preload for a specified tightening torque.

Similarly, the removal torque for a threaded fastener in a bolted joint is defined as

$T_{\mathrm{r}}=F_{\mathrm{p}}\left(-\frac{p}{2 \pi}+\frac{\mu_{\mathrm{t}} r_{\mathrm{t}}}{\cos \beta}+\mu_{\mathrm{n}} r_{\mathrm{n}}\right)$

This removal torque is the torque required to overcome thread and nut friction minus the torque from bolt stretch. Removal torque is the torque applied with a torque wrench to initiate removal or disassembly of a threaded fastener in a bolted joint.

A relation between preload and tightening torque minus removal torque is defined by subtracting $\mathrm{Eq} 4$ from $\mathrm{Eq} 3$ and solving for preload

$F_{\mathrm{p}}=\frac{\pi}{p}\left(T_{\mathrm{t}}-T_{\mathrm{r}}\right)$

This result reveals that preload can be determined from thread pitch, tightening torque and removal torque. In other words, preload can be determined given nothing more than thread pitch and a torque wrench.

For threaded fasteners with a prevailing torque locking feature (e.g., lock nuts and locking inserts), the tightening and removal torque equations become

$T_{\mathrm{t}}=F_{\mathrm{p}}\left(\frac{p}{2 \pi}+\frac{\mu_{\mathrm{t}} r_{\mathrm{t}}}{\cos \beta}+\mu_{\mathrm{n}} r_{\mathrm{n}}\right)+T_{\mathrm{pv}}$

and

$T_{\mathrm{r}}=F_{\mathrm{p}}\left(-\frac{p}{2 \pi}+\frac{\mu_{\mathrm{t}} r_{\mathrm{t}}}{\cos \beta}+\mu_{\mathrm{n}} r_{\mathrm{n}}\right)+T_{\mathrm{pv}}$ 
Here, $T_{\mathrm{pv}}$ is the prevailing torque. It is independent of preload and adds to both tightening and removal torque.

As before, the equation for preload is determined by subtracting removal torque, Eq 7, from tightening torque, Eq 6, and solving for preload to obtain Eq 5. Preload in terms of tightening torque minus removal torque is independent of prevailing torque provided prevailing torque is the same in both directions.

\section{Self-Loosening and Primary Locking}

The idea of obtaining preload from torque difference occurred indirectly. The author had first discovered that self-loosening and primary locking could be determined from tightening and removal torque, then realized preload could be obtained through torque difference. For completeness and further utility, the equations for selfloosening and primary locking are included here.

The negative term in the removal torque equation defines the inherent self-loosening. This results from bolt stretch torque and associated potential energy in the bolt. It is inherent to the threaded fastener and is proportional to preload and thread pitch. This self-loosening can be defined in terms of tightening and removal torque by subtracting Eq 4 from Eq 3 and dividing by 2

$\frac{F_{\mathrm{p}} p}{2 \pi}=\frac{1}{2}\left(T_{\mathrm{t}}-T_{\mathrm{r}}\right)$

The significance of this equation is that self-loosening can be quantified using measurements of tightening torque and removal torque, instead of requiring a measurement of preload [7]. This equation for self-loosening also applies for fasteners with prevailing torque locking as shown by subtracting Eq 7 from Eq 6 and dividing by 2 .

The friction terms in the tightening and removal torque equations define the primary locking in a bolted joint. This primary locking is dependent on preload and friction. The primary locking is defined in terms of tightening and removal torque by adding Eqs 3 and 4 and dividing by 2

$F_{\mathrm{p}}\left(\frac{\mu_{\mathrm{t}} r_{\mathrm{t}}}{\cos \beta}+\mu_{\mathrm{n}} r_{\mathrm{n}}\right)=\frac{1}{2}\left(T_{\mathrm{t}}+T_{\mathrm{r}}\right)$

The significance of this equation is that primary locking can be quantified using measurements of tightening torque and removal torque, instead of requiring estimates of thread interface and nut face coefficients of friction [7].

For threaded fasteners with a prevailing torque locking feature, the primary locking is determined by adding Eqs 6 and 7 and solving for primary locking
$F_{\mathrm{p}}\left(\frac{\mu_{\mathrm{t}} r_{\mathrm{t}}}{\cos \beta}+\mu_{\mathrm{n}} r_{\mathrm{n}}\right)=\frac{1}{2}\left(T_{\mathrm{t}}+T_{\mathrm{r}}\right)-T_{\mathrm{pv}}$

This reveals that even with a prevailing torque locking feature, the primary locking can be quantified with measurements of tightening, removal and prevailing torque.

\section{Uncertainty}

The existing method of using tightening torque control to achieve preload is commonly used but results in significant uncertainty in preload. A primary source for this uncertainty is from thread interface friction and nut face friction (or bolt face friction when using inserts). Uncertainty exists within test samples in torque-tension tests due to variations in test fasteners and interface conditions. Additional variation, not only in fastener and interface conditions but also tools, technique and technician, is introduced for actual fastener hardware used at installation compared to samples used in torque-tension testing. Using this existing method requires previously obtained torque-tension data to specify tightening torque at installation and a specification or previous data for uncertainty.

The new method presented in this paper uses both tightening and removal torque to determine preload. Preload is determined from tightening torque and removal torque measured at installation and thread pitch. No previous torque-tension data are needed for a nut factor to relate tightening torque to preload. Preload can be determined given nothing more than thread pitch and a torque wrench to measure tightening and removal torque at installation.

Since preload is determined by subtracting removal torque from tightening torque, the thread interface friction and nut face friction are subtracted out as shown by Eqs 35. However, subtracting quantities with uncertainty does not usually subtract out the uncertainty. Regardless, uncertainty in preload from torque difference (i.e., tightening minus removal torque) is expected to be less than uncertainty in preload from tightening torque for two reasons.

First, preload from tightening torque alone is based on torque-tension data previously obtained with representative sample fasteners and interface conditions by a different technician, tools and possibly technique than used at installation. Uncertainty results from reproducibility within these previously tested sample fasteners as well as the actual fastener at installation. Preload from torque difference requires no previous data, but instead is based on torque measurements at installation for the actual fastener, interface conditions (including the state of coatings, 
cleanliness and lubricant), technician, technique and tools. Uncertainty results from repeatability (i.e., more than one torque measurement on the actual fastener) rather than reproducibility.

Second, torque wrench accuracy and repeatability are relevant for preload from torque, whereas only torque wrench repeatability is relevant for preload from torque difference. Calibration certificate data generally show accuracy of $2-4 \%$ compared to repeatability of less than $1 \%$.

\section{Testing}

Testing is performed to assess the approach of determining preload from thread pitch, tightening torque and removal torque. The fasteners used include cadmium plated AN414A bolts (0.25-28, 1.53" long) [8], AN315-4 full hex nuts [9], AN365-428A elastic stop lock nuts [10], AN363-428 all-metal lock nuts [11] and AN960-416 washers [12], as well as stainless steel AN4C14A bolts [8], AN315C4R full hex nuts [9], AN363C428 all-metal lock nuts [13] and AN960C416 washers [12].

Figure 1 shows the test equipment and fixture for tests with a bolt and nut. A washer is used under both the bolt head and nut. The fixture integrates a strain gage thru-hole load cell for preload measurement. The load cell is rated for $2000 \mathrm{lb}$ and is $0.63^{\prime \prime}$ high with $2.0^{\prime \prime}$ outer diameter and $0.88^{\prime \prime}$ bottom loading diameter. The accuracy is within $1 \%$ and repeatability is within $\pm 0.1 \%$ full scale. The fixture includes a cone shaped component made of A286 steel, $0.50^{\prime \prime}$ high with $1.1^{\prime \prime}$ top outer diameter, $2.1^{\prime \prime}$ bottom outer diameter and clearance hole for $0.25-28$ bolt. The test fixture with lock nuts is shown in Fig. 2.

In addition, tests with stainless steel Helicoil 11914CN375 non-locking inserts and 3591-4CN375 locking inserts [14] are performed with both AN4-14A and
AN4C14A bolts. Figure 3 shows the test fixture for tests with a bolt and insert. It includes an additional cylindrical shaped bottom component made of A286 steel, 1.1" high with $1.3^{\prime \prime}$ outer diameter and drilled and tapped for $0.25-28$ Helicoil insert installation. A washer is used under the bolt head.

Tightening and removal torque are measured with a dialtype torque wrench. The torque wrench range is $15-75 \mathrm{in}-\mathrm{lb}$ (20-100\% full scale) in increments of 1 in-lb. The accuracy is within $\pm 4 \%$ of torque reading in both directions; however, the actual repeatability from certificate of calibration is within $\pm 1 \%$ of torque reading in both directions. For example, the repeatability at $60 \mathrm{in}-\mathrm{lb}$ is within \pm 0.6 in-lb. In this work, repeatability is more relevant than accuracy since torque difference instead of absolute torque is used to determine preload.

A dial-type torque wrench with a range of 6-30 in-lb (20-100\% full scale) in increments of 0.5 in-lb is used for measuring prevailing torque in tests with lock nuts and locking inserts. The accuracy is $\pm 4 \%$, and repeatability is within $\pm 1 \%$ of torque reading in both directions.

All tests are performed with fasteners in as-received condition. In other words, the fasteners are removed from manufacturer packaging and used without solvent cleaning or addition of lubricant.

Since the recommended range for tightening torque of $\mathrm{AN}-4$ bolts is 50-70 in-lb [15], all test fasteners are assembled using a tightening torque of 60 in-lb plus any measured prevailing torque for lock nuts or locking inserts.

\section{Bolt with Nut Test Data}

Table 1 presents sample data from tests with an AN4C14A bolt and an AN315C4R full hex nut. Each row in the table shows measured data and calculations for a test. For each test, torque is applied to the nut to a tightening torque
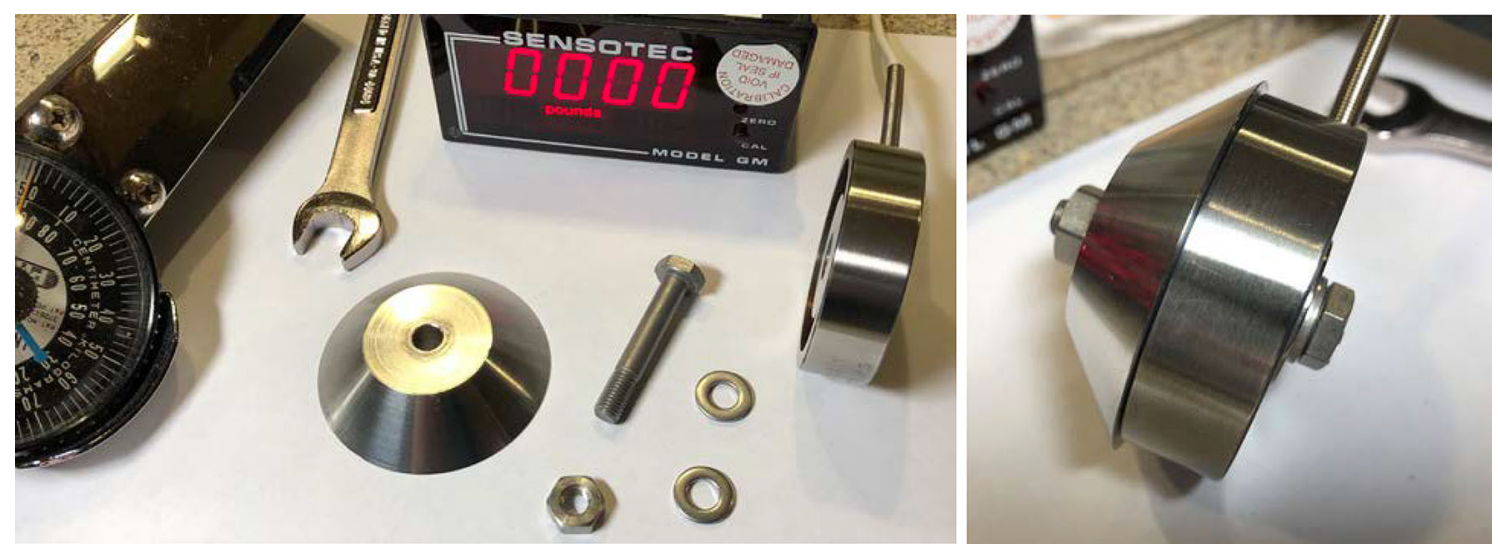

Fig. 1 Test equipment and fixture for bolt with nut tests (full hex nut shown) 


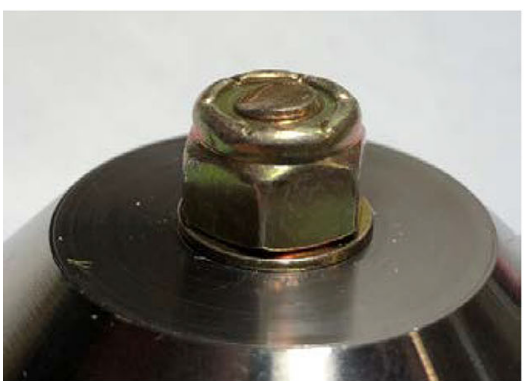

(a)

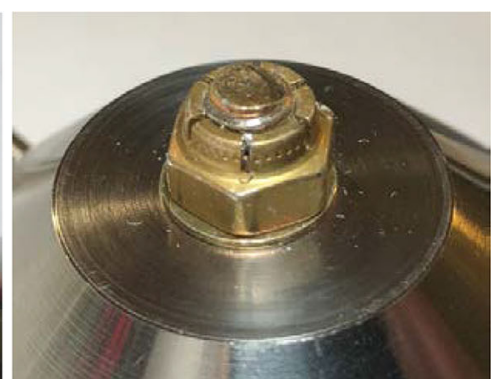

(b)

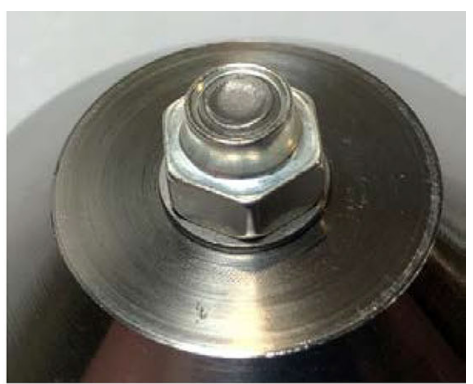

(c)

Fig. 2 Test fixture for bolt with (a) AN365-428A elastic stop lock nut, (b) AN363-428 all-metal lock nut and (c) AN363C428 all-metal lock nut
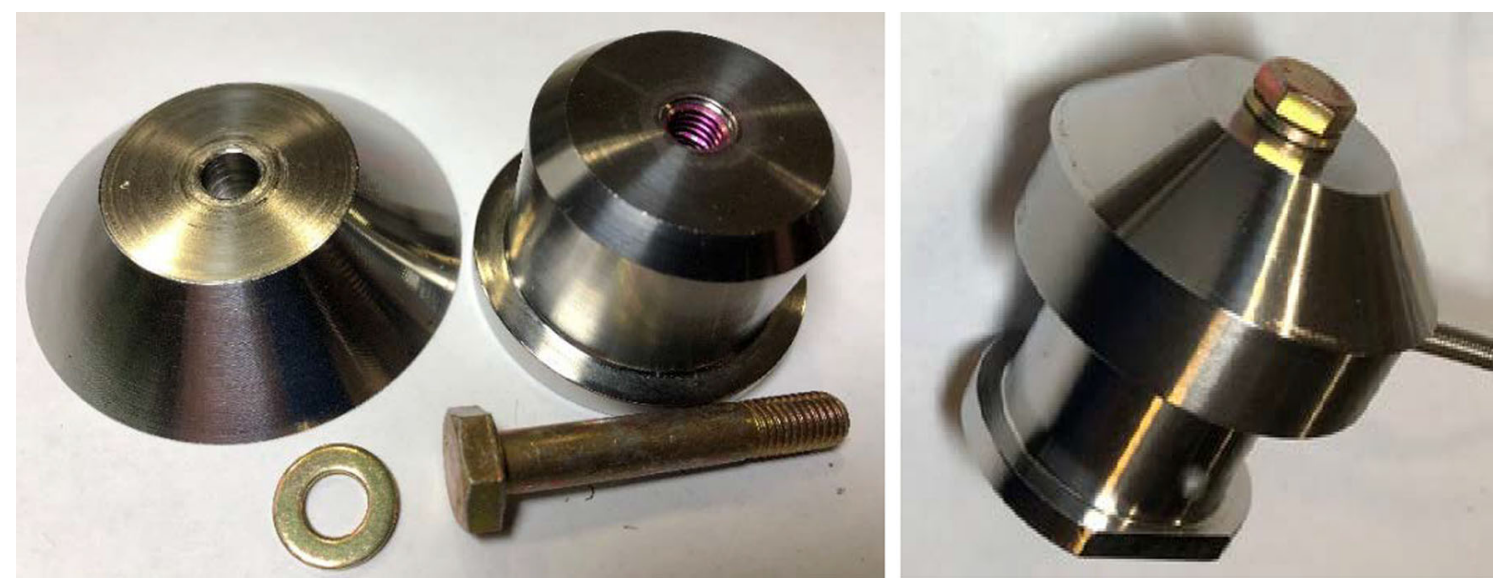

Fig. 3 Test fixture for bolt with insert tests

of $60 \mathrm{in}-\mathrm{lb}$ and the resulting preload measured from the load cell. Then, torque is applied to the nut in the opposite direction to measure removal torque. The preload is calculated from torque difference with $\mathrm{Eq} 5$, and the percent difference in measured and computed preload is calculated. The self-loosening is calculated with $\mathrm{Eq} 8$, first from measured preload and then from measured torque difference. Finally, the primary locking is calculated from torque sum with Eq 9. This is performed for a total of 20 (1 initial use and 19 reuse) tests for the same bolt and nut. Note that the nut was not removed between tests; however, measured preload was reduced to zero before each reuse.

These data show the preload drops with reuse even with the same tightening torque. This is due to an increase in friction at the thread interface and nut face with reuse. This is supported by the computed increase in primary locking with reuse. This is not unexpected since the fasteners used are as-received with no added lubricant.

The self-loosening computed with measured preload compares well with the self-loosening computed with torque difference even with significant reuse. This supports using one-half the tightening minus removal torque for determining self-loosening.
The preload determined with thread pitch and torque difference compares quite well with the measured preload with a maximum percent difference of $12 \%$. Notice that even though the applied tightening torque is constant at 60 in-lb, the measured removal torque increases with reuse and the corresponding computed preload decreases with reuse. Figure 4 plots measured preload against tightening torque for all 20 tests. The spread in measured preload with tightening torque is $315 \mathrm{lb}$. Figure 5 plots measured and computed preload against tightening minus removal torque for all 20 tests. The spread in measured preload for a given torque difference is less than the spread in measured preload with tightening torque alone. Furthermore, the difference between measured preload and computed preload in Fig. 5 is much less than the spread in measured preload with tightening torque in Fig. 4. This supports the use of thread pitch and measured tightening minus removal torque for calculating preload as defined in Eq 5.

A problem with the foregoing approach is that the process of measuring removal torque removes preload. The solution to this problem is to retighten to the tightening torque. Therefore, the process required to use tightening and removal torque to determine preload includes a retighten to the tightening torque. 
Table 1 Measurements and calculations for AN4C14A bolt with AN315C4R full hex nut

\begin{tabular}{|c|c|c|c|c|c|c|c|}
\hline \multicolumn{3}{|c|}{ Measurements } & \multicolumn{5}{|c|}{ Calculations } \\
\hline$T_{\mathrm{t}}(\mathrm{in}-\mathrm{lb})$ & $F_{\mathrm{p}}(\mathrm{lb})$ & $T_{\mathrm{r}}(\mathrm{in}-\mathrm{lb})$ & $\pi\left(T_{\mathrm{t}}-T_{\mathrm{r}}\right) / p(\mathrm{lb})$ & $\%$ diff & $F_{\mathrm{p}} p / 2 \pi(\mathrm{in}-\mathrm{lb})$ & $\left(T_{\mathrm{t}}-T_{\mathrm{r}}\right) / 2(\mathrm{in}-\mathrm{lb})$ & $\left(T_{\mathrm{t}}+T_{\mathrm{r}}\right) / 2($ in-lb $)$ \\
\hline 60 & 1030 & 49 & 968 & 6.1 & 5.9 & 5.5 & 54.5 \\
\hline 60 & 900 & 51 & 792 & 12.0 & 5.1 & 4.5 & 55.5 \\
\hline 60 & 860 & 51 & 792 & 7.9 & 4.9 & 4.5 & 55.5 \\
\hline 60 & 800 & 50 & 880 & -10.0 & 4.5 & 5.0 & 55.0 \\
\hline 60 & 795 & 51 & 792 & 0.4 & 4.5 & 4.5 & 55.5 \\
\hline 60 & 750 & 52 & 704 & 6.2 & 4.3 & 4.0 & 56.0 \\
\hline 60 & 740 & 51 & 792 & -7.0 & 4.2 & 4.5 & 55.5 \\
\hline 60 & 770 & 52 & 704 & 8.6 & 4.4 & 4.0 & 56.0 \\
\hline 60 & 750 & 52 & 704 & 6.2 & 4.3 & 4.0 & 56.0 \\
\hline 60 & 740 & 52 & 704 & 4.9 & 4.2 & 4.0 & 56.0 \\
\hline 60 & 750 & 52 & 704 & 6.2 & 4.3 & 4.0 & 56.0 \\
\hline 60 & 740 & 51 & 792 & -7.0 & 4.2 & 4.5 & 55.5 \\
\hline 60 & 730 & 51 & 792 & -8.4 & 4.1 & 4.5 & 55.5 \\
\hline 60 & 720 & 51 & 792 & -10.0 & 4.1 & 4.5 & 55.5 \\
\hline 60 & 740 & 51 & 792 & -7.0 & 4.2 & 4.5 & 55.5 \\
\hline 60 & 725 & 52 & 704 & 2.9 & 4.1 & 4.0 & 56.0 \\
\hline 60 & 720 & 51 & 792 & -10.0 & 4.1 & 4.5 & 55.5 \\
\hline 60 & 730 & 52 & 704 & 3.6 & 4.1 & 4.0 & 56.0 \\
\hline 60 & 715 & 52 & 704 & 1.6 & 4.1 & 4.0 & 56.0 \\
\hline 60 & 725 & 52 & 704 & 2.9 & 4.1 & 4.0 & 56.0 \\
\hline
\end{tabular}

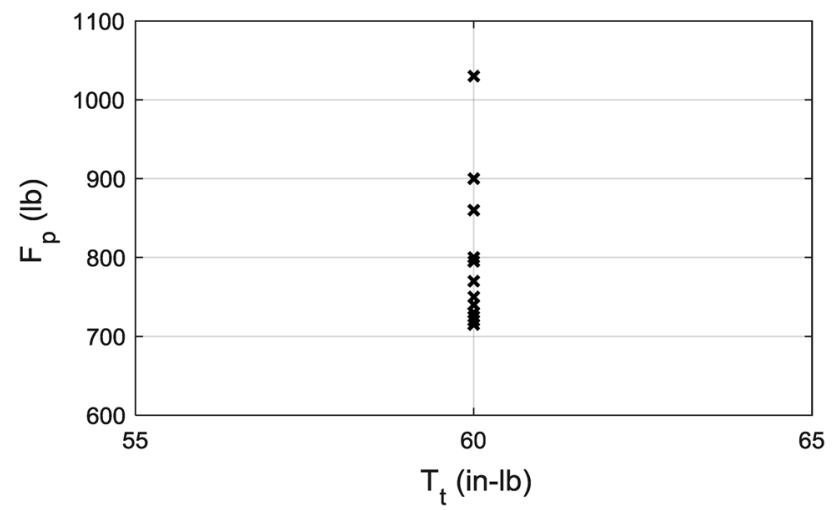

Fig. 4 Preload versus tightening torque for AN4C14A bolt with AN315C4R full hex nut tests

Table 2 presents sample data that includes the retightening to tightening torque and measured preload after this retightening. The process of tighten, removal and retighten is performed 19 times with the same bolt and nut. Each row in Table 2 presents these torque measurements, the preload measurement after retightening, and calculations of preload, percent difference, self-loosening and primary locking for the process. The percent difference shown is between the measured preload after retightening and the computed preload from torque difference.

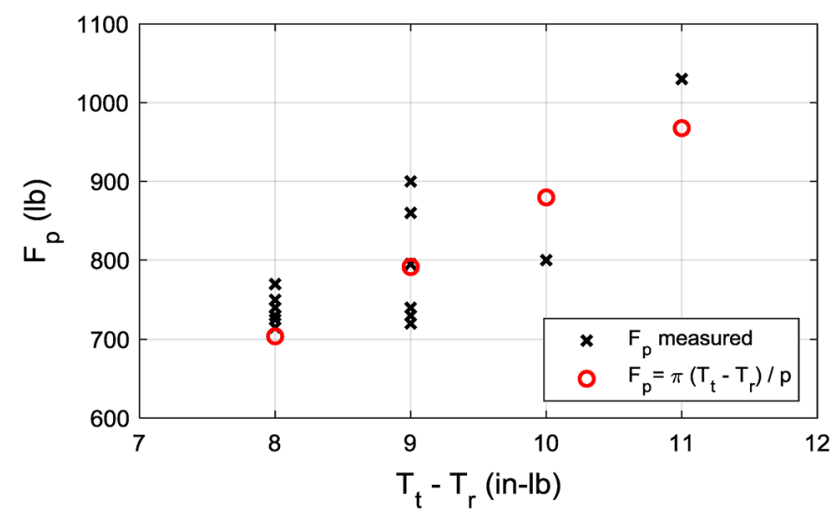

Fig. 5 Preload versus tightening minus removal torque for AN4C14A bolt with AN315C4R full hex nut tests

The preload determined with thread pitch and torque difference compares very well with the measured preload after retightening with a maximum percent difference of $-10.6 \%$. Figure 6 plots measured preload after retightening against tightening torque for all 19 preload measurements. The spread in measured preload is $185 \mathrm{lb}$. Figure 7 plots this measured preload and corresponding computed preload against tightening minus removal torque. The difference between computed preload and measured preload after retightening in Fig. 7 is much less than the spread in measured preload after retightening with 
Table 2 Measurements and calculations for AN4C14A bolt with AN315C4R full hex nut with retightening

\begin{tabular}{|c|c|c|c|c|c|c|c|c|}
\hline \multicolumn{4}{|c|}{ Measurements } & \multicolumn{5}{|c|}{ Calculations } \\
\hline$T_{\mathrm{t}}(\mathrm{in}-\mathrm{lb})$ & $T_{\mathrm{r}}$ (in-lb) & $T_{\mathrm{t}}(\mathrm{in}-\mathrm{lb})$ & $F_{\mathrm{p}}(\mathrm{lb})$ & $\pi\left(T_{\mathrm{t}}-T_{\mathrm{r}}\right) / p(\mathrm{lb})$ & $\%$ diff & $F_{\mathrm{p}} p / 2 \pi(\mathrm{in}-\mathrm{lb})$ & $\left(T_{\mathrm{t}}-T_{\mathrm{r}}\right) / 2(\mathrm{in}-\mathrm{lb})$ & $\left(T_{\mathrm{t}}+T_{\mathrm{r}}\right) / 2$ (in-lb) \\
\hline 60 & 49 & 60 & 900 & 968 & -7.5 & 5.1 & 5.5 & 54.5 \\
\hline 60 & 51 & 60 & 860 & 792 & 7.9 & 4.9 & 4.5 & 55.5 \\
\hline 60 & 51 & 60 & 800 & 792 & 1.0 & 4.5 & 4.5 & 55.5 \\
\hline 60 & 50 & 60 & 795 & 880 & -10.6 & 4.5 & 5.0 & 55.0 \\
\hline 60 & 51 & 60 & 750 & 792 & -5.6 & 4.3 & 4.5 & 55.5 \\
\hline 60 & 52 & 60 & 740 & 704 & 4.9 & 4.2 & 4.0 & 56.0 \\
\hline 60 & 51 & 60 & 770 & 792 & -2.8 & 4.4 & 4.5 & 55.5 \\
\hline 60 & 52 & 60 & 750 & 704 & 6.2 & 4.3 & 4.0 & 56.0 \\
\hline 60 & 52 & 60 & 740 & 704 & 4.9 & 4.2 & 4.0 & 56.0 \\
\hline 60 & 52 & 60 & 750 & 704 & 6.2 & 4.3 & 4.0 & 56.0 \\
\hline 60 & 52 & 60 & 740 & 704 & 4.9 & 4.2 & 4.0 & 56.0 \\
\hline 60 & 51 & 60 & 730 & 792 & -8.4 & 4.1 & 4.5 & 55.5 \\
\hline 60 & 51 & 60 & 720 & 792 & -10.0 & 4.1 & 4.5 & 55.5 \\
\hline 60 & 51 & 60 & 740 & 792 & -7.0 & 4.2 & 4.5 & 55.5 \\
\hline 60 & 51 & 60 & 725 & 792 & -9.2 & 4.1 & 4.5 & 55.5 \\
\hline 60 & 52 & 60 & 720 & 704 & 2.3 & 4.1 & 4.0 & 56.0 \\
\hline 60 & 51 & 60 & 730 & 792 & -8.4 & 4.1 & 4.5 & 55.5 \\
\hline 60 & 52 & 60 & 715 & 704 & 1.6 & 4.1 & 4.0 & 56.0 \\
\hline 60 & 52 & 60 & 725 & 704 & 2.9 & 4.1 & 4.0 & 56.0 \\
\hline
\end{tabular}

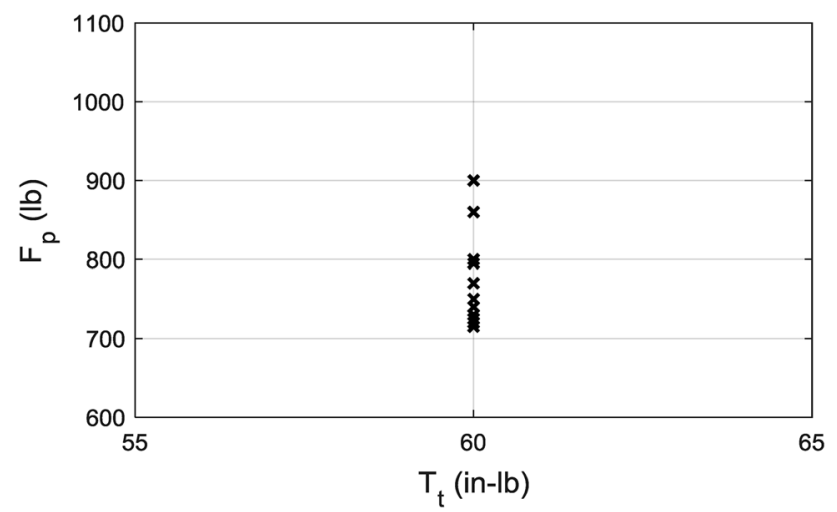

Fig. 6 Preload versus tightening torque for AN4C14A bolt with AN315C4R full hex nut tests using measured preload after retightening

tightening torque in Fig. 6. This supports the use of thread pitch and measured tightening minus removal torque for calculating preload as defined in Eq 5 with retightening to tightening torque included in the process.

As an example of using this approach, consider a 0.25 28 bolt and nut installed with a torque wrench to a tightening torque of $60 \mathrm{in}-\mathrm{lb}$. The torque wrench is used to measure the removal torque of $49 \mathrm{in}-\mathrm{lb}$ and then used to retighten to $60 \mathrm{in}-\mathrm{lb}$. The preload is calculated using Eq 5

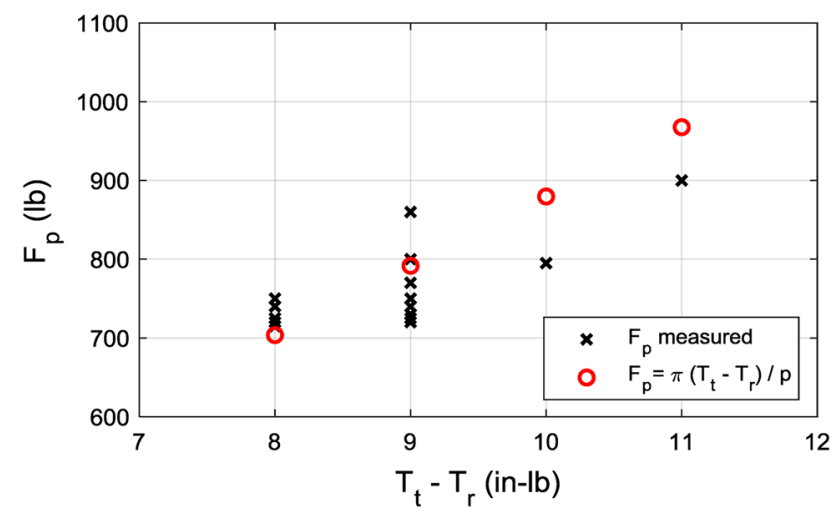

Fig. 7 Preload versus tightening minus removal torque for AN4C14A bolt with AN315C4R full hex nut tests using measured preload after retightening

$F_{\mathrm{p}}=\frac{\pi}{p}\left(T_{\mathrm{t}}-T_{\mathrm{r}}\right)=\frac{\pi}{(1 / 28)}(60-49)=968 \mathrm{lb}$

Since the data used in this example are from row 1 of Table 2, the measured preload from retightening is $900 \mathrm{lb}$. The percent difference between measured and computed preload is $-7.5 \%$. From Eq 8, the self-loosening computed from measured torque difference compares well to the self-loosening computed from measured preload after retightening 
Table 3 Measurements and calculations for AN4-14A bolt with AN365-428A lock nut

\begin{tabular}{|c|c|c|c|c|c|c|c|c|c|c|c|}
\hline \multicolumn{7}{|c|}{ Measurements } & \multicolumn{5}{|c|}{ Calculations } \\
\hline $\begin{array}{l}T_{\mathrm{pv} \text { on }} \\
\text { (in-lb) }\end{array}$ & $\begin{array}{c}T_{\mathrm{t}} \\
\text { (in-lb) }\end{array}$ & $\begin{array}{c}T_{\mathrm{r}} \\
\text { (in-lb) }\end{array}$ & $\begin{array}{l}T_{\mathrm{pv} \text { off }} \\
\text { (in-lb) }\end{array}$ & $\begin{array}{l}T_{\mathrm{pv} \text { on }} \\
\text { (in-lb) }\end{array}$ & $\begin{array}{c}T_{\mathrm{t}} \\
\text { (in-lb) }\end{array}$ & $\begin{array}{c}F_{\mathrm{p}} \\
(\mathrm{lb})\end{array}$ & $\begin{array}{c}\pi\left(T_{\mathrm{t}}-T_{\mathrm{r}}\right) / p \\
(\mathrm{lb})\end{array}$ & $\begin{array}{c}\% \\
\text { diff }\end{array}$ & $\begin{array}{c}F_{\mathrm{p}} p / 2 \pi \\
\text { (in-lb) }\end{array}$ & $\begin{array}{l}\left(T_{\mathrm{t}}-T_{\mathrm{r}}\right) / 2 \\
\quad(\mathrm{in}-\mathrm{lb})\end{array}$ & $\begin{array}{c}\left(T_{\mathrm{t}}+T_{\mathrm{r}}\right) / 2-T_{\mathrm{pv}} \\
(\mathrm{in}-\mathrm{lb})\end{array}$ \\
\hline 15 & 75 & 64 & 12 & 14 & 74 & 979 & 880 & 10.1 & 5.6 & 5.0 & 55.0 \\
\hline 14 & 74 & 62 & 12 & 13 & 73 & 940 & 968 & -2.9 & 5.3 & 5.5 & 54.5 \\
\hline 13 & 73 & 63 & 12 & 12 & 72 & 893 & 792 & 11.3 & 5.1 & 4.5 & 55.5 \\
\hline 12 & 72 & 62 & 12 & 12 & 72 & 852 & 880 & -3.2 & 4.8 & 5.0 & 55.0 \\
\hline 12 & 72 & 62 & 12 & 12 & 72 & 850 & 880 & -3.5 & 4.8 & 5.0 & 55.0 \\
\hline 12 & 72 & 63 & 12 & 13 & 73 & 809 & 880 & -8.7 & 4.6 & 5.0 & 55.0 \\
\hline 13 & 73 & 63 & 13 & 12 & 72 & 824 & 792 & 3.9 & 4.7 & 4.5 & 55.5 \\
\hline 12 & 72 & 63 & 12 & 12 & 72 & 751 & 792 & -5.4 & 4.3 & 4.5 & 55.5 \\
\hline 12 & 72 & 64 & 12 & 12 & 72 & 736 & 704 & 4.4 & 4.2 & 4.0 & 56.0 \\
\hline 12 & 72 & 64 & 12 & 12 & 72 & 667 & 704 & -5.5 & 3.8 & 4.0 & 56.0 \\
\hline 12 & 72 & 64 & 12 & 12 & 72 & 681 & 704 & -3.3 & 3.9 & 4.0 & 56.0 \\
\hline
\end{tabular}

$$
\begin{aligned}
\frac{1}{2}\left(T_{\mathrm{t}}-T_{\mathrm{r}}\right) & =\frac{1}{2}(60-49)=5.5 \mathrm{in} \mathrm{lb} \\
\frac{F_{p} p}{2 \pi} & =\frac{(900)(1 / 28)}{2 \pi}=5.1 \mathrm{in} \mathrm{lb}
\end{aligned}
$$

And from Eq 9, the primary locking is computed from torque sum as

$$
\begin{aligned}
F_{\mathrm{p}}\left(\frac{\mu_{\mathrm{t}} r_{\mathrm{t}}}{\cos \beta}+\mu_{\mathrm{n}} r_{\mathrm{n}}\right) & =\frac{1}{2}\left(T_{\mathrm{t}}+T_{\mathrm{r}}\right)=\frac{1}{2}(60+49) \\
& =54.5 \mathrm{in} \mathrm{lb}
\end{aligned}
$$

\section{Bolt with Lock Nut Test Data}

Table 3 presents sample data from tests with an AN4-14A bolt and an AN365-428A elastic stop lock nut. In these tests, the 6-30 in-lb range torque wrench is used on the lock nut to measure the "on" prevailing torque in the tightening direction once the locking feature is fully engaged with zero preload. The 15-75 in-lb range torque wrench is then used on the lock nut to apply a tightening torque of 60 in-lb plus the measured prevailing torque. For example, if the measured prevailing torque is $15 \mathrm{in}-\mathrm{lb}$, then the tightening torque is 75 in-lb. Next, torque is applied to the lock nut in the opposite direction to measure the removal torque. The lock nut is turned until there is zero preload with full engagement of locking feature; then, the "off" prevailing torque is measured. The lock nut is retightened, first measuring the "on" prevailing torque and then applying a tightening torque of 60 in-lb plus the prevailing torque. The preload is measured with the load cell after retightening.

The process of tighten, removal and retighten is performed 11 times with the same bolt and lock nut. Each row in Table 3 presents torque measurements, preload measurement after retighten, and calculations of preload, percent difference, self-loosening and primary locking for the process. The percent difference shown is between the measured preload after retightening and the computed preload from torque difference.

These data show a decrease in "on" prevailing torque with reuse. This is an expected result with lock nuts $[16,17]$. The "off" prevailing torque is less than the "on" prevailing torque for the initial use and first couple reuses, but then is consistent in both directions. If significantly different in both directions, adjustments should be made in calculating preload and self-loosening. The tightening torque is 60 in-lb plus the "on" prevailing torque. As in the previous data, the preload drops with reuse due to an increase in friction at the thread interface and nut face which is supported by the computed increase in primary locking with reuse. This is not unexpected since the fasteners are used as-received with no added lubricant.

The self-loosening computed with torque difference compares quite well with the self-loosening computed with measured preload after retightening. This supports using torque difference for determining self-loosening even for a lock nut with reuse.

The preload determined with thread pitch and torque difference compares quite well with the measured preload after retightening with a maximum percent difference of $11.3 \%$. Figure 8 plots measured preload after retightening against tightening torque minus prevailing torque. The spread in measured preload is $312 \mathrm{lb}$. Figure 9 plots the corresponding measured and computed preload against tightening minus removal torque. The difference between computed preload and measured preload after retightening in Fig. 9 is much less than the spread in measured preload with tightening torque minus prevailing torque in Fig. 8. This supports the calculation of preload with thread pitch 


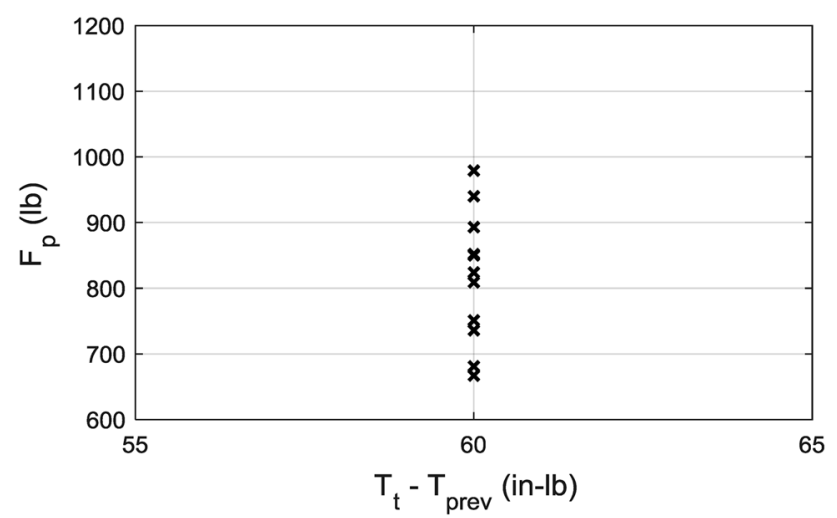

Fig. 8 Preload versus tightening torque minus prevailing torque for AN4-14A bolt with AN365-428A lock nut tests

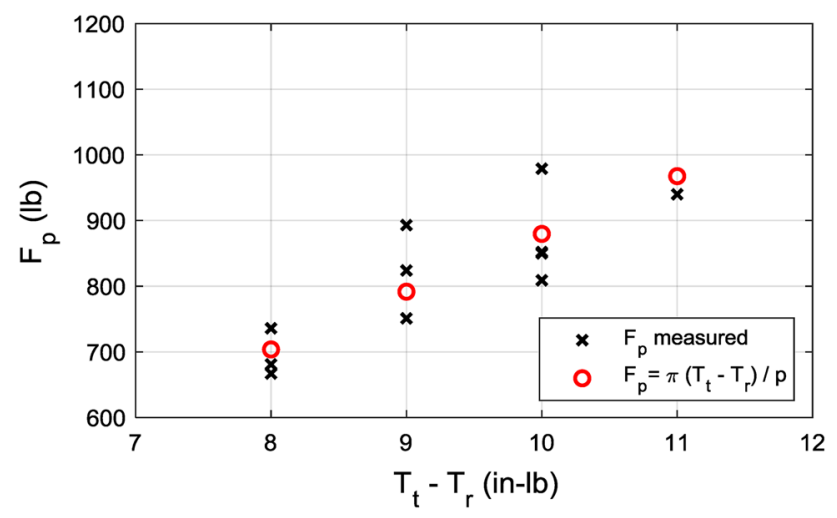

Fig. 9 Preload versus tightening minus removal torque for AN4-14A bolt with AN365-428A lock nut tests

and measured tightening minus removal torque even for a lock nut with a prevailing torque locking feature and reuse.

\section{Bolt with Locking Insert Test Data}

Table 4 presents sample data from tests with an AN4-14A bolt and a 3591-4CN375 locking Helicoil insert. In these tests, the torque wrench is applied to the bolt head. As before, the lower range torque wrench is used to measure the prevailing torque in both the "on" and "off" directions under the conditions of fully engaged locking feature and zero preload. The higher range torque wrench is used to apply a tightening and retightening torque of 60 in-lb plus the measured "on" prevailing torque and to measure removal torque. The preload after retightening is measured with the load cell.

The process of tighten, removal and retighten is performed 11 times with the same bolt and locking insert. Each row in Table 4 presents the torque measurements, preload measurement after retighten, and calculations of preload, percent difference, self-loosening and primary locking for the process. The percent difference shown is between the measured preload after retightening and the computed preload from torque difference.

These data show a significant decrease in prevailing torque with reuse. This is an expected result with locking inserts [16]. As in the previous data, the preload drops with reuse due to an increase in friction at the thread interface and bolt head face as indicated by the increase in computed primary locking.

The self-loosening computed with measured preload after retightening compares well with the self-loosening computed with torque difference. This supports using onehalf the tightening minus removal torque for determining self-loosening for bolts even with locking inserts and reuse.

The preload determined with thread pitch and torque difference compares well with the measured preload after

Table 4 Measurements and calculations for AN4C14A bolt with 3591-4CN375 Helicoil insert

\begin{tabular}{|c|c|c|c|c|c|c|c|c|c|c|c|}
\hline \multicolumn{7}{|c|}{ Measurements } & \multicolumn{5}{|c|}{ Calculations } \\
\hline $\begin{array}{l}T_{\mathrm{pv} \text { on }} \\
\text { (in-lb) }\end{array}$ & $\begin{array}{c}T_{\mathrm{t}} \\
\text { (in-lb) }\end{array}$ & $\begin{array}{c}T_{\mathrm{r}} \\
\text { (in-lb) }\end{array}$ & $\begin{array}{l}T_{\mathrm{pv} \text { off }} \\
\text { (in-lb) }\end{array}$ & $\begin{array}{l}T_{\mathrm{pv} \text { on }} \\
\text { (in-lb) }\end{array}$ & $\begin{array}{c}T_{\mathrm{t}} \\
\text { (in-lb) }\end{array}$ & $\begin{array}{l}F_{\mathrm{p}} \\
(\mathrm{lb})\end{array}$ & $\begin{array}{c}\pi\left(T_{\mathrm{t}}-T_{\mathrm{r}}\right) / p \\
(\mathrm{lb})\end{array}$ & $\%$ diff & $\begin{array}{c}F_{\mathrm{p}} p / 2 \pi \\
\text { (in-lb) }\end{array}$ & $\begin{array}{l}\left(T_{\mathrm{t}}-T_{\mathrm{r}}\right) / 2 \\
\quad(\mathrm{in}-\mathrm{lb})\end{array}$ & $\begin{array}{c}\left(T_{\mathrm{t}}+T_{\mathrm{r}}\right) / 2-T_{\mathrm{pv}} \\
\text { (in-lb) }\end{array}$ \\
\hline 15 & 75 & 58 & 15 & 15 & 75 & 1332 & 1495 & -12.3 & 7.6 & 8.5 & 51.5 \\
\hline 15 & 75 & 59 & 11 & 11 & 71 & 1240 & 1056 & 14.9 & 7.0 & 6.0 & 54.0 \\
\hline 11 & 71 & 57 & 8 & 8 & 68 & 1131 & 968 & 14.4 & 6.4 & 5.5 & 54.5 \\
\hline 8 & 68 & 54 & 8 & 8 & 68 & 1125 & 1232 & -9.5 & 6.4 & 7.0 & 53.0 \\
\hline 8 & 68 & 54 & 6 & 6 & 66 & 1002 & 1056 & -5.3 & 5.7 & 6.0 & 54.0 \\
\hline 6 & 66 & 55 & 6 & 6 & 66 & 991 & 968 & 2.4 & 5.6 & 5.5 & 54.5 \\
\hline 6 & 66 & 55 & 6 & 6 & 66 & 997 & 968 & 2.9 & 5.7 & 5.5 & 54.5 \\
\hline 6 & 66 & 55 & 6 & 6 & 66 & 959 & 968 & -0.9 & 5.5 & 5.5 & 54.5 \\
\hline 6 & 66 & 55 & 6 & 6 & 65 & 885 & 880 & 0.6 & 5.0 & 5.0 & 54.5 \\
\hline 6 & 65 & 55 & 6 & 6 & 65 & 864 & 880 & -1.8 & 4.9 & 5.0 & 54.5 \\
\hline 6 & 65 & 55 & 6 & 6 & 65 & 851 & 880 & -3.4 & 4.8 & 5.0 & 54.5 \\
\hline
\end{tabular}




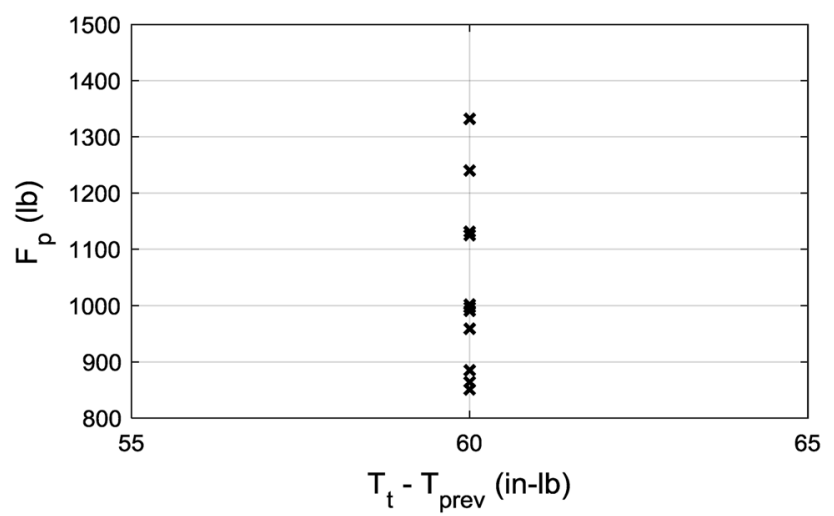

Fig. 10 Preload versus tightening torque minus prevailing torque for AN4C14A bolt with 3591-4CN375 Helicoil insert tests

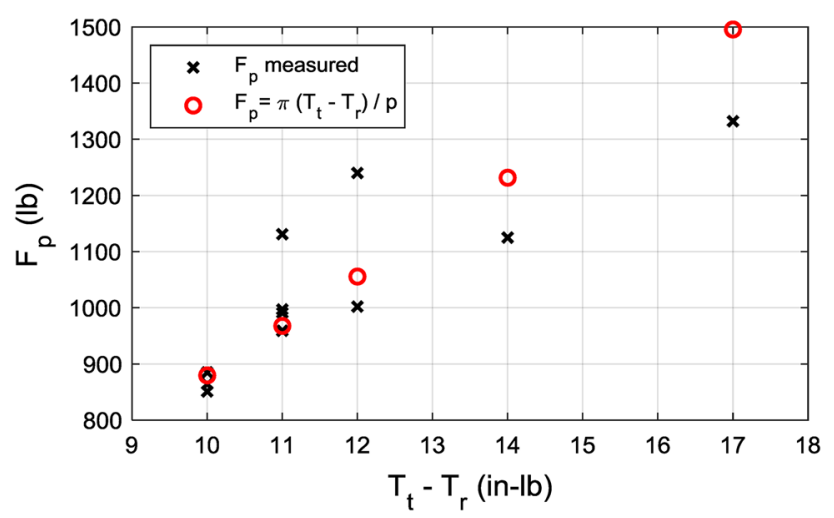

Fig. 11 Preload versus tightening minus removal torque for AN4C14A bolt with 3591-4CN375 Helicoil insert tests

retightening with a maximum percent difference of $14.9 \%$. Figure 10 plots measured preload after retightening against tightening torque minus prevailing torque. The spread in measured preload is $481 \mathrm{lb}$. Figure 11 plots the measured and computed preload against tightening minus removal torque. The difference between computed preload and measured preload after retightening in Fig. 11 is much less than the spread in measured preload in Fig. 10. This supports the calculation of preload with thread pitch and measured tightening minus removal torque even for bolts with locking inserts and reuse.

As an example of using this approach for fasteners with a prevailing torque locking feature, consider a $0.25-28$ bolt with a locking insert. The bolt is installed into the insert with torque wrenches with a measured "on" prevailing torque of $15 \mathrm{in}-\mathrm{lb}$ and tightening torque of $75 \mathrm{in}-\mathrm{lb}$. Torque wrenches are used to measure a removal torque of $58 \mathrm{in}-\mathrm{lb}$ and an "off" prevailing torque of 15 in-lb. Then, the bolt is retightened with a measured "on" prevailing torque of 15 in-lb and tightening torque of $75 \mathrm{in}-\mathrm{lb}$. The preload is calculated using Eq 5.
$F_{\mathrm{p}}=\frac{\pi}{p}\left(T_{\mathrm{t}}-T_{\mathrm{r}}\right)=\frac{\pi}{(1 / 28)}(75-58)=1495 \mathrm{lb}$

Since the data used in this example are from row 1 of Table 4, the measured preload after retightening is $1332 \mathrm{lb}$. The percent difference between measured and computed preload is $-12.3 \%$. From Eq 8, the self-loosening computed from measured torque difference compares well to the self-loosening computed from measured preload after retightening

$$
\begin{aligned}
\frac{1}{2}\left(T_{\mathrm{t}}-T_{\mathrm{r}}\right) & =\frac{1}{2}(75-58)=8.5 \mathrm{in} \mathrm{lb} \\
\frac{F_{\mathrm{p}} p}{2 \pi} & =\frac{(1332)(1 / 28)}{2 \pi}=7.6 \mathrm{in} \mathrm{lb}
\end{aligned}
$$

And from Eq 10, the primary locking is computed as

$$
\begin{aligned}
F_{\mathrm{p}}\left(\frac{\mu_{\mathrm{t}} r_{\mathrm{t}}}{\cos \beta}+\mu_{\mathrm{n}} r_{\mathrm{n}}\right) & =\frac{1}{2}\left(T_{\mathrm{t}}+T_{\mathrm{r}}\right)-T_{\mathrm{pv}}=\frac{1}{2}(75+58)-15 \\
& =51.5 \mathrm{in} \mathrm{lb}
\end{aligned}
$$

Similar data were obtained for the other fasteners tested. The samples presented are representative in showing reduced uncertainty in preload from tightening minus removal torque versus just tightening torque. However, tests with the stainless steel AN4C14A bolts and AN363C428 all-metal locknuts resulted in severe galling and seizing during the initial use and were not tested further.

All tests performed to date have been with fasteners in the as-received condition with no cleaning performed or lubricant added. Since the addition of lubricant to the threads and nut face (or bolt face for use with inserts) improves repeatability and reduces uncertainty in preload in torque-tension data, it is expected that the addition of lubricant will reduce the uncertainty in computed preload from torque difference as well.

\section{Application}

The approach presented in this paper provides a way to determine preload from nothing more than thread pitch, tightening and removal torque. No additional information or previous data are needed. The tightening and removal torque can be measured with a common torque wrench at point of use.

Consider the following example. Given a torque wrench and fasteners (e.g., a bolt and nut) with a thread pitch of 1/ $28^{\prime \prime}$, tighten the fasteners to a preload of $900 \mathrm{lb}$. The desired preload can be achieved by simply trying a few values of tightening torque, measuring the associated removal torque and computing the preload using Eq 5. 
Table 5 Preload from only thread pitch, tightening and removal torque

\begin{tabular}{lcc}
\hline$T_{\mathrm{t}}$ (in-lb) & $T_{\mathrm{r}}(\mathrm{in}-\mathrm{lb})$ & $\pi\left(T_{\mathrm{t}}-T_{\mathrm{r}}\right) / p(\mathrm{lb})$ \\
\hline 20 & 16 & 352 \\
30 & 24 & 528 \\
40 & 32 & 703 \\
50 & 40 & 879 \\
60 & 49 & 967 \\
\hline
\end{tabular}

Table 5 presents actual data following this process. An initial trial tightening torque is $20 \mathrm{in}-\mathrm{lb}$. This results in a trial removal torque of $16 \mathrm{in}-\mathrm{lb}$ and a computed trial preload of $352 \mathrm{lb}$. The tightening torque can be incrementally increased until the desired preload is achieved. The results in Table 5 indicate a tightening torque of $52 \mathrm{in}-\mathrm{lb}$ should provide close to the desired preload of $900 \mathrm{lb}$. The actual preload was measured and was within a few percent.

The needed tightening torque can be determined even quicker by comparing the $352 \mathrm{lb}$ preload achieved with the initial trial $20 \mathrm{in}-\mathrm{lb}$ tightening torque with the desired preload of $900 \mathrm{lb}$. A simple ratio of $T_{\mathrm{t}} / T_{\mathrm{t}}$ trial $=F_{\mathrm{p}} / F_{\mathrm{p} \text { trial }}$ gives

$T_{\mathrm{t}}=\frac{T_{\mathrm{t} \text { trial }} F_{\mathrm{p}}}{F_{\mathrm{p} \text { trial }}}=\frac{p T_{\mathrm{t} \text { trial }} F_{\mathrm{p}}}{\pi\left(T_{\mathrm{t} \text { trial }}-T_{\mathrm{r} \text { trial }}\right)}$

This equation provides a required tightening torque of (20)(900)/352 = 51 in-lb which will achieve the desired preload within a few percent. The entire process to achieve the desired preload is to apply a trial tightening torque, measure removal torque and calculate and apply tightening torque for desired preload using Eq 17.

This approach works even if the trial tightening torque is too high. For example, using the data in Table 5, if the trial tightening torque is $60 \mathrm{in}-\mathrm{lb}$, the measured removal torque is $49 \mathrm{in}-\mathrm{lb}$ and the computed trial preload is $967 \mathrm{lb}$. Using Eq 17, the tightening torque required for the desired preload is (60)(900)/967 = 56 in-lb which will achieve the desired preload within a few percent.

\section{Variations and Technique}

The approach presented in this paper for achieving preload using both tightening and removal torque measurements with a torque wrench is an improvement over the existing method of using only tightening torque. The new approach requires no previously acquired torque-tension data and provides preload with reduced uncertainty. Additional expensive tools, training and preparation are not needed as with other existing methods based on turn angle or bolt stretch. Other than thread pitch, no previous knowledge, data or preparation is required. Because the new approach is based on tightening minus removal torque measurements at installation, it inherently accommodates variations in thread interface and nut or bolt face conditions including the state of coatings, cleanliness and lubricant.

Similarly, the new approach accommodates variations in technique with the torque wrench. For example, some technicians prefer to apply torque slowly and hold at the tightening torque, while others promote a more rapid application approach. Such differences can be quite significant if using just tightening torque based on previously obtained torque-tension data. The technician or user at installation needs to be aware of which technique was used in obtaining the torque-tension data. With the new approach, application of torque should be as consistent as possible for tightening and removal torque measurements. A slow steady application of torque for tightening with a couple second hold at tightening torque is recommended and was used in this work. Too rapid application of tightening torque may result in lower friction compared to the friction associated with removal torque. Any difference in friction between tightening and removal likely accounts for most of the uncertainty in using this new approach to achieve preload.

\section{Conclusions}

This paper presented a novel approach to determine preload at installation or point of use with a common torque wrench. The approach uses both tightening torque and removal torque. This work revealed preload is proportional to tightening minus removal torque and that taking this torque difference subtracts out friction. Tests performed with bolts, nuts, lock nuts, inserts and locking inserts to assess this method of determining preload showed good agreement with measured preload. The tests performed found the uncertainty in preload from torque difference to be less than the uncertainty in preload from just tightening torque alone.

A useful takeaway application from this work is that a desired preload with reduced uncertainty can be achieved with nothing more than knowledge of thread pitch and a torque wrench to measure tightening and removal torque. This was supported with example data and calculations. The significance of this application is that preload is determined without any previously acquired torque-tension data as is needed with tightening torque alone for achieving preload.

In addition to developing an equation and presenting supporting test data for preload in terms of tightening and removal torque, this paper developed equations and presented supporting data for self-loosening and primary 
locking of threaded fasteners in terms of tightening and removal torque. Self-loosening can be quantified from torque measurements alone instead of requiring a measurement of preload to calculate it. Primary locking can be quantified from torque measurements alone instead of requiring knowledge of thread interface friction and nut or bolt face friction in addition to preload. Self-loosening, primary locking and preload can be determined or verified at point-of-use installation with a common torque wrench.

Additional testing to assess preload from tightening minus removal torque is planned for larger samples of fasteners and conditions including use of lubricants. This will allow a broader assessment of uncertainty. The application procedure presented for determining desired preload from nothing more than thread pitch and trial tightening and removal torque measurements will be further tested for a wider range of conditions.

\section{References}

1. MIL-HDBK-60, Threaded fasteners: tightening to proper tension (Department of Defense Handbook, 2006)

2. NASA-STD-5020A, Requirements for threaded fastening systems in spaceflight hardware (2018)

3. VDI 2230, Systematic calculation of highly stressed bolted joints-joints with one cylindrical bolt (Verein Deutscher Ingenieure, Düsseldorf, 2015)
4. NASM1312-15 Rev 1, Fastener test methods: torque-tension (National Aerospace Standard, 2012)

5. E. Oberg, F. Jones, H. Horton, H. Ryffel, Machinery's Handbook, 25th edn. (Industrial Press, Norwalk, 1996), p. 1408

6. J. Bickford, An Introduction to the Design and Behavior of Bolted Joints (CRC Press, Boca Raton, 1995)

7. D. Hess, Threaded fastener secondary locking requirements. J. Fail. Anal. Prev. 17, 724-730 (2017)

8. AN3 THRU AN20, Bolt-machine, aircraft (Department of Defense Military Specification Sheet, 1991)

9. NASM315, Nut, plain, hexagon, airframe (National Aerospace Standard, 2002)

10. NASM21044, Nut, self-locking, hexagon, regular height, 250F, $125 \mathrm{ksi}$ ftu and $60 \mathrm{ksi}$ ftu (National Aerospace Standard, 1999)

11. NASM21045, Nut, self-locking, hexagon, regular height, 450F, 125 ksi ftu (National Aerospace Standard, 1999)

12. NAS1149, Washer, flat (National Aerospace Standard, 2019)

13. NASM21046, Nut, self-locking, hexagon, regular height, 800F, $125 \mathrm{ksi}$ ftu (National Aerospace Standard, 2000)

14. NASM8846, Inserts, screw-thread, helical coil (National Aerospace Standard, 1998)

15. AC 43.13-1B, Acceptable methods, techniques, and practices: aircraft inspection and repair (Federal Aviation Administration, 1998)

16. A. Zimandy, D. Hess, Effect of reuse of locknuts with prevailing torque locking feature. J. Fail. Anal. Prev. 13, 121-127 (2013)

17. D. Hess, Prevailing torque locking in threaded fastener. J. Fail. Anal. Prev. 18, 1562-1572 (2018)

Publisher's Note Springer Nature remains neutral with regard to jurisdictional claims in published maps and institutional affiliations. 